\title{
Durvalumab after concurrent chemoradiotherapy in a patient with chemotherapy-resistant unresectable stage III non-small cell lung cancer: a case report
}

\author{
Tao Zhang ${ }^{1 \wedge}$, Yuqi Wu ${ }^{1}$, Shan Zheng ${ }^{2}$, Guowei Cheng ${ }^{3}$, Xiangling $\mathrm{He}^{3}$, Nan $\mathrm{Bi}^{1}$ \\ ${ }^{1}$ Department of Radiation Oncology, ${ }^{2}$ Department of Pathology, National Cancer Center/National Clinical Research Center for Cancer/Cancer \\ Hospital, Chinese Academy of Medical Science and Peking Union Medical College, Beijing, China; ${ }^{3}$ Department of Radiation Oncology, Cancer \\ Hospital of HuanXing ChaoYang District Beijing, Beijing, China \\ Correspondence to: Nan Bi. Department of Radiation Oncology, National Cancer Center/National Clinical Research Center for Cancer/Cancer \\ Hospital, Chinese Academy of Medical Science and Peking Union Medical College, No. 17 Panjiayuan Nanli, Chaoyang District, Beijing 100021, \\ China. Email: binan_email@163.com.
}

\begin{abstract}
Durvalumab as consolidative immunotherapy following concurrent chemoradiotherapy (cCRT) is the standard treatment for patients with unresectable stage III non-small cell lung cancer (NSCLC). Here, we describe a case of unresectable stage III NSCLC who was chemotherapy resistant, benefit from cCRT and durvalumab. Contrast-enhanced computed tomography (CT) evaluation of the patient detected a mass in the left upper mediastinum. The biopsy specimen showed evidence of poorly differentiated pulmonary carcinoma. Brain magnetic resonance imaging (MRI) and bone scan found no evidence of distant metastasis. The clinical stage was defined as T4N2M0 IIIB. The patient underwent one cycle of induction chemotherapy with cisplatin and etoposide. Positron-emission tomography/computed tomography (PET-CT) after induction chemotherapy showed the tumor increased in size. The patient received volumetric modulated arc therapy (VMAT) with simultaneous integrated boost (SIB) and the radiation dose was 60.2 Gy/2.15 Gy/28 F. Concurrent chemotherapy was delivered and the regimen was weekly paclitaxel liposome combined with nedaplatin. Durvalumab was given one day after the final fraction of radiotherapy. CT evaluation performed one month and three months after treatment found partial response (PR). Grade 1 pneumonitis was reported as a toxicity. The use of durvalumab following cCRT was feasible and efficient in this patient who was resistant to chemotherapy and that its early use was safe.
\end{abstract}

Keywords: Non-small cell lung cancer (NSCLC); concurrent chemoradiotherapy (cCRT); durvalumab; chemotherapy resistance; case report

Submitted Apr 23, 2020. Accepted for publication Jul 04, 2020.

doi: 10.21037/apm-20-1118

View this article at: http://dx.doi.org/10.21037/apm-20-1118

\section{Introduction}

Before the PACIFIC trial, the standard treatment for unresectable stage III non-small cell lung cancer (NSCLC) was concurrent chemoradiotherapy (cCRT). RTOG 9410 (1) confirmed that cCRT with cisplatin-based chemotherapy achieved long-term survival benefit compared with sequential chemoradiotherapy (sCRT). Nevertheless, less than $50 \%$ of patients actually received cCRT; in the majority of cases, patients with unresectable stage III NSCLC first received chemotherapy, which reduced the chance of them receiving cCRT. In fact, the CALGB 39801 study (2) showed that there was no improvement in survival when induction chemotherapy was used before

$\wedge$ ORCID: 0000-0003-4861-0679 


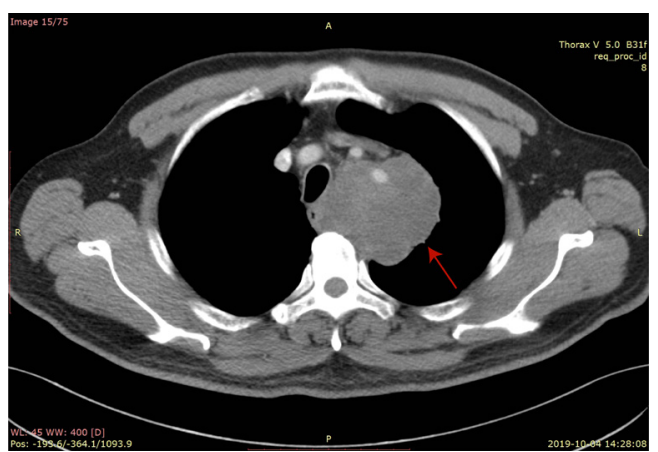

Figure 1 CT image at baseline. Arrow showed the mass in the left upper mediastinum. CT, computed tomography.

cCRT. RTOG 0617 (3) found standard-dose (60 Gy) radiotherapy with concurrent chemotherapy improved overall survival compared with high-dose radiotherapy (74 Gy) with concurrent chemotherapy. As consolidation chemotherapy $(4,5)$ did not improve survival when added to cCRT, cCRT alone with 60 Gy radiation dose should remained the standard of care. After the PACIFIC trial was reported (6-8), cCRT followed by durvalumab as consolidation immunotherapy became the standard treatment for patients with unresectable stage III NSCLC. However, some institutions continued to use induction chemotherapy. In the CALGB 39801 study (2), after induction chemotherapy, $39 \%$ patients had stable disease (SD) and 6\% patients had progressive disease (PD), which indicates that almost half of the patients do not benefit from induction chemotherapy. We reported the outcome of a patient with unresectable stage III NSLCLC who received cCRT followed by durvalumab after achieving SD after induction chemotherapy. We present the following case in accordance with the CARE reporting checklist (available at http://dx.doi.org/10.21037/apm-20-1118).

\section{Case presentation}

A 63-year-old man presented to the hospital with cough and blood-tinged sputum in October, 2019. The patient had a 40 -year history of smoking 2 packs/day. One year before, he had been diagnosed with diabetes and had a family history of gastric cancer. Contrast-enhanced computed tomography (CT) evaluation of the chest detected a mass (with the diameter of the largest section measuring $7.7 \mathrm{~cm} \times$ $6.7 \mathrm{~cm}$ ) in the left upper mediastinum, surrounding the left subclavian artery (Figure 1). The initial evaluation found compression of the bronchi and the esophagus, as well as lymphadenopathy. Chronic bronchitis, emphysema, and pulmonary bullae in both lungs were also observed. Endobronchial ultrasound-guided transbronchial needle aspiration (EBUS-TBNA) was performed. The biopsy specimen of the left upper paratracheal lymph node (2L) showed evidence of poorly differentiated pulmonary carcinoma. The results of immunohistochemical staining indicated high-grade neuroendocrine cancer, but were limited by sample size (Figure 2). Immunohistochemical staining results: AE1/AE3 (3+), CK18 (1+), CK7 (-), CD5/6 (-), TTF-1 (1+), NapsinA (-), ChrA (-), Syno (1+), CD56 (-), P63 (-), P40 (-), LCA (-), Ki-67 (+, 40\%), PD-1 (+, $5 \%$ in lymphocytes), PD-L1 (22C3) (tumor proportion score, TPS: $<1 \%)$. Brain magnetic resonance imaging (MRI) and bone scan found no evidence of distant metastasis. Lung cancer (poorly differentiated carcinoma) was diagnosed. The clinical stage was T4N2M0 IIIB, as defined by American Joint Committee on Cancer (AJCC) eighth edition.

The patient then underwent one cycle of chemotherapy with cisplatin and etoposide from Oct 26, 2019 to Oct 28, 2019. The response was evaluated by positron-emission tomography/computed tomography (PET-CT). The diameter of the largest section of the mass had increased to $9.0 \mathrm{~cm} \times 9.0 \mathrm{~cm}$. The patient's response was evaluated as SD (Figure 3A). Uptake of 18F-fluorodeoxyglucose (18F-FDG) was detected in the mass, with a maximum standard uptake value (SUVmax) of 8.2 (Figure 3B). Multiple small lymph nodes were detected in the mediastinum $(2 \mathrm{R}, 4 \mathrm{R}, 4 \mathrm{~L}, 5)$ and both hilars had slight FDG uptake (SUVmax 2.3).

As the tumor was enlarged in size, external-beam radiation therapy with concurrent chemotherapy was administered over the next six weeks. Volumetric modulated arc therapy (VMAT) (9) with simultaneous integrated boost (SIB) (10) was delivered by Elekta (VersaHD, Sweden) from November 25, 2019 to January 3, 2020 (Figure 4). Gross tumor volume (GTV) was defined as the tumor in the left upper mediastinum. The lymph node in the mediastinum (2L) was melted with the primary tumor and included in the GTV. GTV of lymph nodes (GTVnd) was defined as the volume of the suspected lymph nodes in the mediastinum (4L/5). Clinical target volume (CTV) was defined as the GTV with a $6 \mathrm{~mm}$ expansion, the GTVnd with a $5 \mathrm{~mm}$ expansion, and the regions of $2 \mathrm{~L}, 4 \mathrm{~L} / 5$. CTV was defined based on the principles of involved-field RT (11). Planning target volume (PTV) was defined as CTV with a $5-\mathrm{mm}$ margin. Planning GTV (PGTV) was defined as the sum of GTV and GTVnd with a 5-mm expansion; 95\% of PGTV received $60.2 \mathrm{~Gy} / 2.15 \mathrm{~Gy} / 28 \mathrm{~F}$ and $95 \%$ of PTV received 

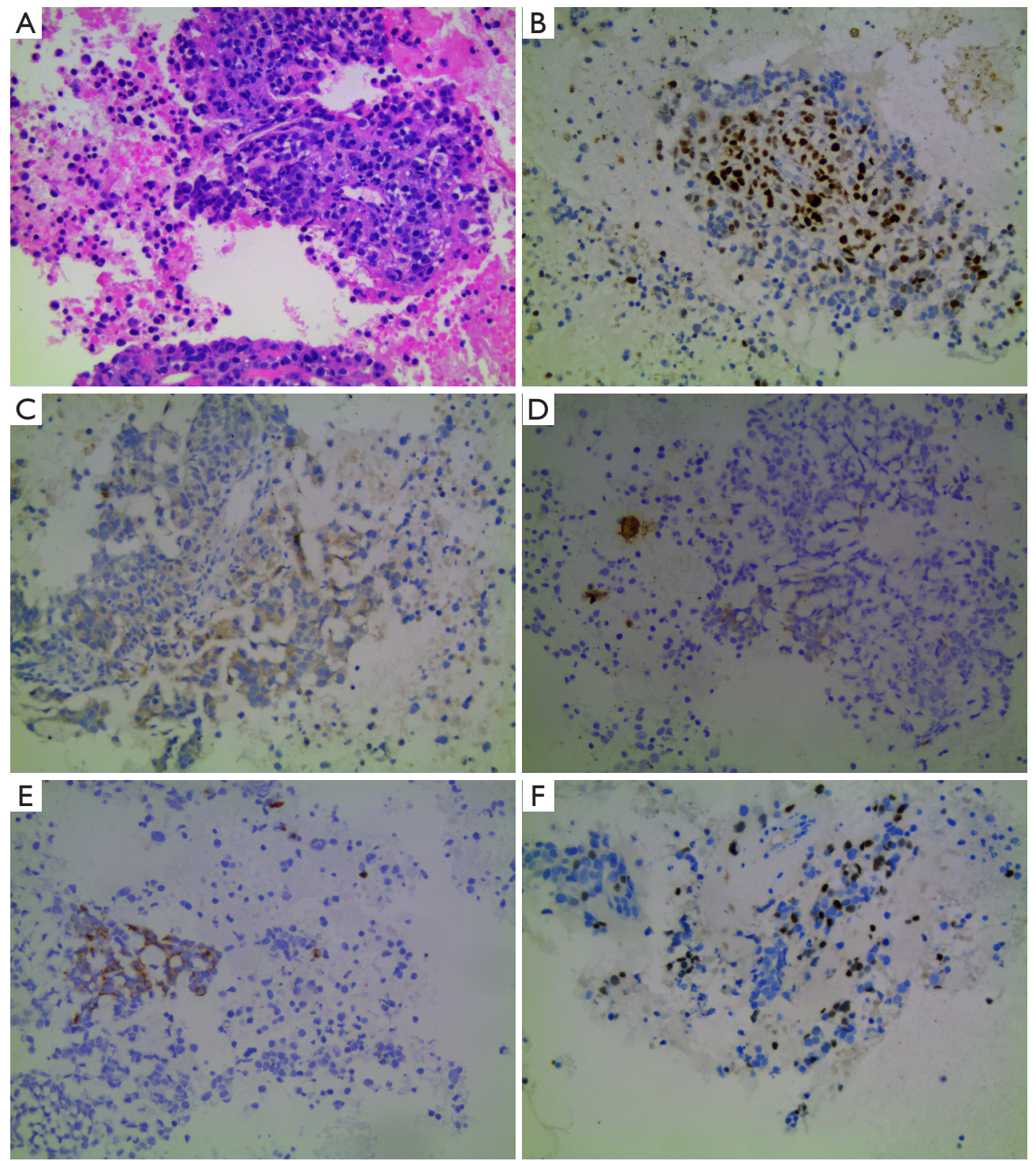

Figure 2 Pathological images of the biopsy specimen from the left upper paratracheal lymph node. (A) HE staining, $\times 200$; (B) Ki67 staining, $\times 200$; (C) Syno staining, ×200; (D) AE1/AE3 staining, ×200; (E) CK18 staining, ×200; (F) TTF-1 staining, ×200.

50.4 Gy/1.8 Gy/28 F. The mean dose to the lungs (MLD) was $9.8 \mathrm{~Gy}$. The percentage of lung volume that received a dose in excess of 5 Gy (V5), 20 Gy (V20), and 30 Gy (V30) was $35.7 \%, 18.0 \%$, and $13.0 \%$, respectively. Concurrent chemotherapy was delivered and the regimen was adjusted to weekly paclitaxel liposome combined with nedaplatin. In total, four cycles of concurrent chemotherapy were completed. The toxicities reported during cCRT included a decrease in white blood cells (grade II) and nausea (grade II), based on the Common Terminology Criteria for Adverse Events (CTCAE) 4.0 criteria.
One day after cCRT, the patient immediately received durvalumab $(10 \mathrm{mg} / \mathrm{kg}, \mathrm{q} 14 \mathrm{~d})$ as consolidation therapy. A day after the first infusion, he developed a transient fever, from which he self-recovered. During the third cycle, elevated erythrocyte sedimentation rates (ESR) $(93 \mathrm{~mm}$ $1^{\text {st }}$ hour$)$ and C-reactive protein (CRP) $(2.26 \mathrm{mg} / \mathrm{dL})$ were detected. A repeat CT scan obtained four weeks after radiotherapy (2 cycles of durvalumab) was completed showed an obvious decrease in the size of the primary tumor $(5.3 \mathrm{~cm} \times 4.3 \mathrm{~cm})$ (Figure 5). The patient's response was evaluated based on the Response Evaluation Criteria 

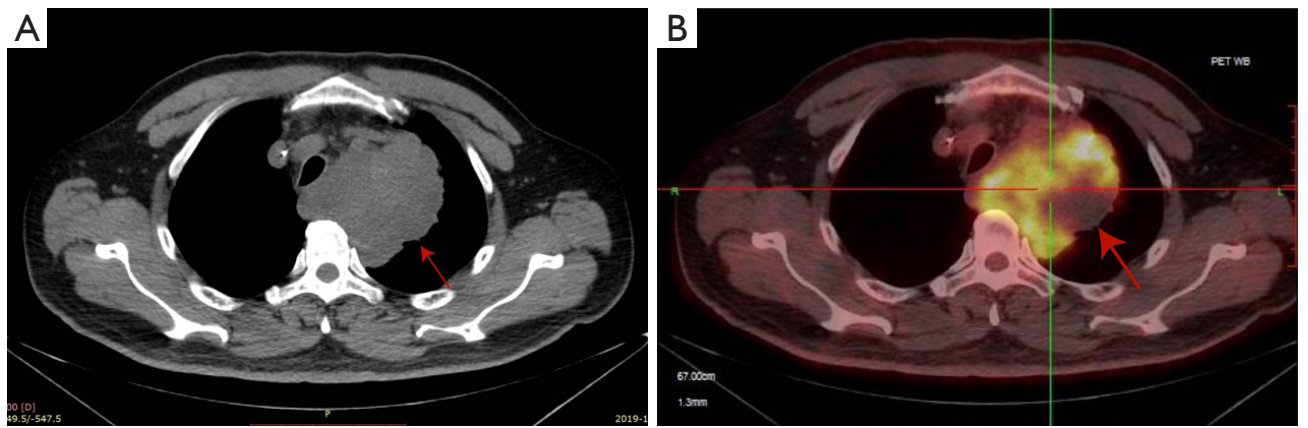

Figure 3 CT and PET-CT images after one cycle of induction chemotherapy (before cCRT): (A) CT image and (B) PET-CT image. CT, computed tomography; PET, positron-emission tomography; cCRT, concurrent chemoradiotherapy. Arrow showed the mass in in the left upper mediastinum, which increased in size.

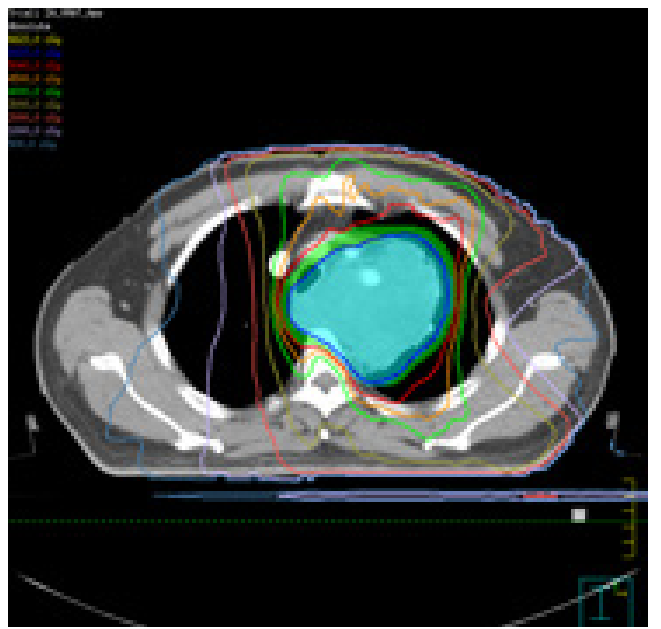

Figure 4 Radiotherapy volumes and dose distribution; the green area represents PTV and the sky blue area represents PGTV. PTV, planning target volume; PGTV, planning gross tumor volume.

in Solid Tumors (RECIST) version 1.1 and showed partial response (PR). A routine follow-up CT scan three months after CRT showed grade 1 pneumonitis but no evidence of clinical progression or relapse of cancer (Figure 6). The tumor had decreased in size to $4.6 \mathrm{~cm} \times 3.4 \mathrm{~cm}$. The patient reported no other toxicities. The patient continued to receive durvalumab as consolidative therapy every 2 weeks due to the good response and tolerable toxicity. The patient and his family expressed that the effect of cCRT followed by durvalumab exceeded their expectations and they would continue and finish this consolidative treatment.

All procedures performed in this case involving human participants were in accordance with the Declaration of Helsinki (as revised in 2013). Written informed consent was



Figure 5 CT image from one month after cCRT. CT, computed tomography; cCRT, concurrent chemoradiotherapy. Arrow showed the mass in the left upper mediastinum decreased in size.

obtained from the patient for publication of this manuscript and any accompanying images.

\section{Discussion}

According to Global Cancer Statistics (GLOBOCAN) estimation, in 2018, lung cancer was the most common and deadly cancer in the world (12). For patients with unresectable locally advanced NSCLC, cCRT followed by durvalumab is the standard treatment, which can improve progression-free survival (PFS) [hazard ratio $(\mathrm{HR})=0.52$, $95 \%$ confidence interval $(\mathrm{CI}): 0.42-0.65, \mathrm{P}<0.001]$ and overall survival $(\mathrm{OS})(\mathrm{HR}=0.68,95 \% \mathrm{CI}: 0.53-0.87$, $\mathrm{P}<0.001)(2,3)$.

Durvalumab is a selective, high-affinity, human IgG1 monoclonal antibody, that binds to programmed death ligand 1 (PD-L1) and blocks its interaction with programmed death 1 (PD-1) and cluster of differentiation 

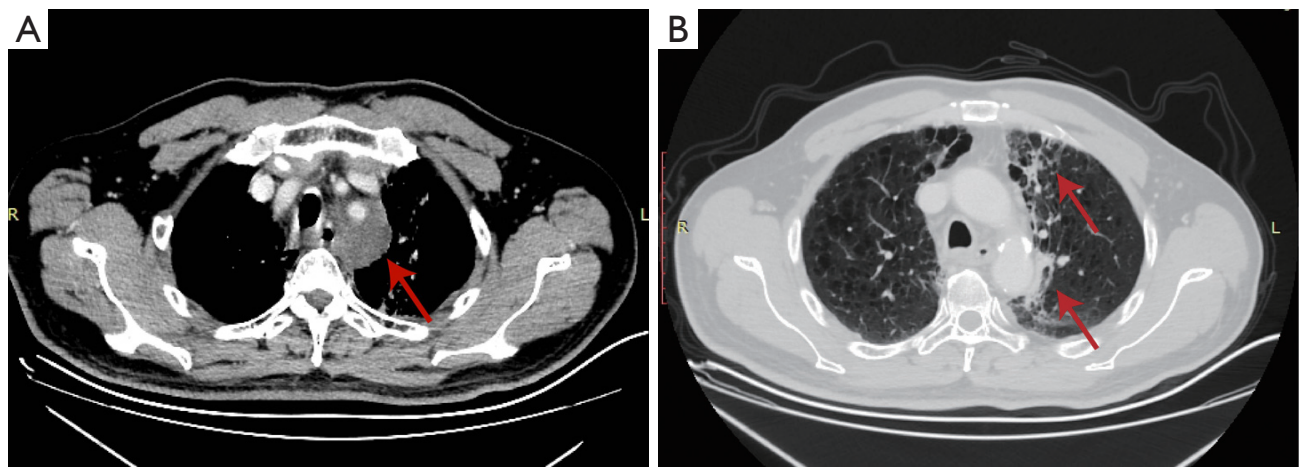

Figure 6 CT images from three months after cCRT: (A) the mediastinal window, arrow showed the mass in the left upper mediastinum; (B) the lung window, arrow showed grade 1 pneumonitis. CT, computed tomography; cCRT, concurrent chemoradiotherapy.

(CD)80 (B7.1). PD-L1 is expressed in a broad range of cancers. The binding of durvalumab to the PD-L1 receptor inhibits the interaction of PD-L1 with the PD-1 and CD80 receptors expressed on immune cells and allows $\mathrm{T}$ cells to recognize and kill tumor cells (7).

Based on subgroup analysis of PACIFIC, patients in the $<14$ days from last radiation to randomization subgroup had the lower HR (95\% CI: 0.28-0.66), which suggests that early consolidation immunotherapy may carry more benefit for patients (8). As we known, radiotherapy can increase antigen visibility of tumor, modulates the tumor microenvironment and regulate immune checkpoint expression, which can increase the effect of immunotherapy. And immunotherapy can sensitize tumors to radiation (13). According to this, our patient received his first cycle of durvalumab as early as possible (one day after final radiotherapy). After two cycles of durvalumab, evaluation showed that the tumor size had clearly decreased, which means that immunotherapy and RT enhanced the effect of each other.

In this case, the patient started immunotherapy very early and experienced no severe adverse events, which shows that durvalumab can be used safely soon after cCRT. Transient fever and pneumonitis experienced by the patient were classified as grade 1 according to the CTC AE 4.0 criteria. From the data of PACIFIC trial (7), the use of durvalumab as consolidative immunotherapy does not increase the risk of any toxicity or pneumonitis. The rates of grade 3 and 4 pneumonitis or radiation pneumonitis were $3.4 \%$ in the study group and $2.6 \%$ in the control group, respectively. The real world data from a French study (14) was consistent with the results of the PACIFIC trial: the rate of toxicity was low, and the rate of durvalumab-related pneumonitis was $1.1 \%$.

From this case, we found that the use of durvalumab following cCRT was feasible in a patient who was resistant to chemotherapy and that its early use was efficient and safe.

\section{Acknowledgments}

Funding: This work was supported by CAMS Innovation Fund for Medical Sciences (2017-I2M-1-009).

\section{Footnote}

Reporting Checklist: The authors have completed the CARE reporting checklist. Available at http://dx.doi.org/10.21037/ apm-20-1118

Conflicts of Interest: All authors have completed the ICMJE uniform disclosure form (available at http://dx.doi. org/10.21037/apm-20-1118). The authors have no conflicts of interest to declare.

Etbical Statement: The authors are accountable for all aspects of the work in ensuring that questions related to the accuracy or integrity of any part of the work are appropriately investigated and resolved. All procedures performed in this case involving human participants were in accordance with the Declaration of Helsinki (as revised in 2013). Written informed consent was obtained from the patient for publication of this manuscript and any accompanying images.

Open Access Statement: This is an Open Access article distributed in accordance with the Creative Commons 
Attribution-NonCommercial-NoDerivs 4.0 International License (CC BY-NC-ND 4.0), which permits the noncommercial replication and distribution of the article with the strict proviso that no changes or edits are made and the original work is properly cited (including links to both the formal publication through the relevant DOI and the license). See: https://creativecommons.org/licenses/by-nc-nd/4.0/.

\section{References}

1. Curran WJ Jr, Paulus R, Langer CJ, et al. Sequential vs. concurrent chemoradiation for stage III non-small cell lung cancer: randomized phase III trial RTOG 9410. J Natl Cancer Inst 2011;103:1452-60.

2. Vokes EE, Herndon JE 2nd, Kelley MJ, et al. Induction chemotherapy followed by chemoradiotherapy compared with chemoradiotherapy alone for regionally advanced unresectable stage III Non-small-cell lung cancer: Cancer and Leukemia Group B. J Clin Oncol 2007;25:1698-704.

3. Bradley JD, Hu C, Komaki RR, et al. Long-Term Results of NRG Oncology RTOG 0617: Standard- Versus HighDose Chemoradiotherapy With or Without Cetuximab for Unresectable Stage III Non-Small-Cell Lung Cancer. J Clin Oncol 2020;38:706-14.

4. Ahn JS, Ahn YC, Kim JH, et al. Multinational Randomized Phase III Trial With or Without Consolidation Chemotherapy Using Docetaxel and Cisplatin After Concurrent Chemoradiation in Inoperable Stage III NonSmall-Cell Lung Cancer: KCSG-LU05-04. J Clin Oncol 2015;33:2660-6.

5. Hanna N, Neubauer M, Yiannoutsos C, et al. Phase III study of cisplatin, etoposide, and concurrent chest radiation with or without consolidation docetaxel in patients with inoperable stage III non-small-cell lung cancer: the Hoosier Oncology Group and U.S. Oncology. J Clin Oncol 2008;26:5755-60.

6. Antonia SJ, Villegas A, Daniel D, et al. Overall Survival

Cite this article as: Zhang T, Wu Y, Zheng S, Cheng G, He $\mathrm{X}$, Bi N. Durvalumab after concurrent chemoradiotherapy in a patient with chemotherapy-resistant unresectable stage III non-small cell lung cancer: a case report. Ann Palliat Med 2020;9(4):2375-2380. doi: 10.21037/apm-20-1118 with Durvalumab after Chemoradiotherapy in Stage III NSCLC. N Engl J Med 2018;379:2342-50.

7. Dempke WC, Fenchel K. Durvalumab for non-resectable stage IIIB non-small cell lung cancer-a small step or a big leap? Transl Lung Cancer Res 2018;7:S153-7.

8. Gray JE, Villegas A, Daniel D, et al. Three-Year Overall Survival with Durvalumab after Chemoradiotherapy in Stage III NSCLC-Update from PACIFIC. J Thorac Oncol 2020;15:288-93.

9. Wu K, Xu X, Li X, et al. Radiation pneumonitis in lung cancer treated with volumetric modulated arc therapy. J Thorac Dis 2018;10:6531-9.

10. Wang D, Bi N, Zhang T, et al. Comparison of efficacy and safety between simultaneous integrated boost intensitymodulated radiotherapy and conventional intensitymodulated radiotherapy in locally advanced non-smallcell lung cancer: a retrospective study. Radiat Oncol 2019;14:106.

11. Chen M, Bao Y, Ma HL, et al. Involved-field radiotherapy versus elective nodal irradiation in combination with concurrent chemotherapy for locally advanced non-small cell lung cancer: a prospective randomized study. Biomed Res Int 2013;2013:371819.

12. Bray F, Ferlay J, Soerjomataram I, et al. Global cancer statistics 2018: GLOBOCAN estimates of incidence and mortality worldwide for 36 cancers in 185 countries. CA Cancer J Clin 2018;68:394-424.

13. Wang Y, Liu ZG, Yuan H, et al. The Reciprocity between Radiotherapy and Cancer Immunotherapy. Clin Cancer Res 2019;25:1709-17.

14. Avrillon V, Bota Ouchlif S, Merle P, et al. 1470P - First real life data on durvalumab after definitive concomitant chemoradiotherapy (cCRT) in unresectable stage (St) III non-small cell lung cancer (NSCLC) in France: Analysis of 591 patients (pts) enrolled in the French cohort (c) temporary authorization of use (ATU). Ann Oncol 2019;30:v597. 